\title{
REFORÇO DIDATICO SOBRE TRIGONOMETRIA, USANDO MICROCOMPUTADOR
}

Amēlia Quites Antoniazzi; Maria do Carmo Argemi Abreu e Maria Friguetto Mezzomo

Departamento de Matemātica. Centro de Ciências Naturais e Exatas. UFSM. Santa Maria, RS.

RESUMO

0 objetivo deste aplicativo é proporcionar ao aluno da 2 a sērie do 20 grau, um reforço do conteúdo de trigonometria. Este pro grama compreende um estudo sobre razões trigonométricas num triângu 10 retāngulo, razões trigonomētricas num triângulo qualquer, trigo nometria na circunferência e ciclo trigonométrico. Foi utilizado no desenvolvimento do programa, um microcomputador da linha Prológica, 64 kb, 16 bits, Linguagem BASIC.

\section{SUMMARY}

ANTONIAZZI, A.Q.; ABREU, M.C.A. and MEZZOMO, M.F., 1989. Didatic reinforcement about trigonometry using microcomputer. Ciên cia e Natura 11: .27-36, 1989

The objective of this work is to provide the high school student attending second grade, a reinforcement of the content of trigonometry. This program includes a study about trigonometry reason in a any triangle trigonometry in the circunference, and trigonometric cicle. In the development of the program, is was used on IBM-PC mi crocomputer.

INTRODUÇÃO

Buscando formas alternativas para o estudo da matemātica do 20 grau, elaborou-se um aplicativo que dê ao aluno mais uma opção de estudo, como reforço didātico.

Este aplicativo foi desenvolvido com a orientação de uma professora da 2a série do 20 grau, de acordo com os conteúdos minis trados em aula, iniciando-se o programa com uma revisão teórica, que serve como apoio para os exercicios apresentados a seguir.

DESCRIÇÃO DO APLICATIVO

Tendo por base o conteúdo ministrado em sala de aula pela professora da $2 \stackrel{a}{-}$ sērie do 20 grau, elaborou-se o seguinte programa:

a) Diālogo inicial entre o microcomputador e o aluno;

b) Introdução e definições gerais sobre trigonometria;

c) Resumo teōrico e exercỉcios, com respostas sobre: c. 1 - razões trigonomētricas num triângulo retângulo. 
c.2 - razões trigonométricas num triângulo qualquer.

c.3 - trigonometria na circunferēncia.

c.4 - ciclo trigonométrico.

d) Diālogo final entre o microcomputador e o aluno.

\section{CONSIDERAÇOES FINAIS}

Após o término do estudo por parte dos alunos, faz-se uma análise dos resultados obtidos, em conjunto com a professora da $2^{a}$ série do 20 grau, sobre o rendimento dos mesmos na referida discipli na.

\section{B IBL IOGRAF IA}

CASTRO, Cláudio de M. O Computador na escola. Rio de Janeiro, Campus, 1988.

CHAVES, Eduardo O.C. \& SETZER, Waldemar W. O uso de computadores em escolas. São Paulo, Scipione, 1988.

FALKEMBACH, Gilse M. Linguagem BASIC. Polïgrafo, s.ed. s.d.

GIOVANNI, José R. \& BonjoRno, José R. Matemática 2 e grau. São Paulo, FTD, 1979.

IEZZI, Gelson \& MURAKAMI, Carlos. Fundamentos de matemática elemen tar. vol. III, São Paulo, Atual, 1981.

NETO, Aref Antor \& LAPA, Nilton. Trigonometria. São Paulo, Moderna, 1979.

NETTO, Scipione \& GOES, Célia. Matemática, processo auto-instrutivo. São Paulo, Scipione, 1977.

STEINBRUCK, Marỉlia. Introdução à Linguagem BASIC. Porto Alegre, Sa gra, 1981.

\section{LISTAGEM DO PROGRAMA}

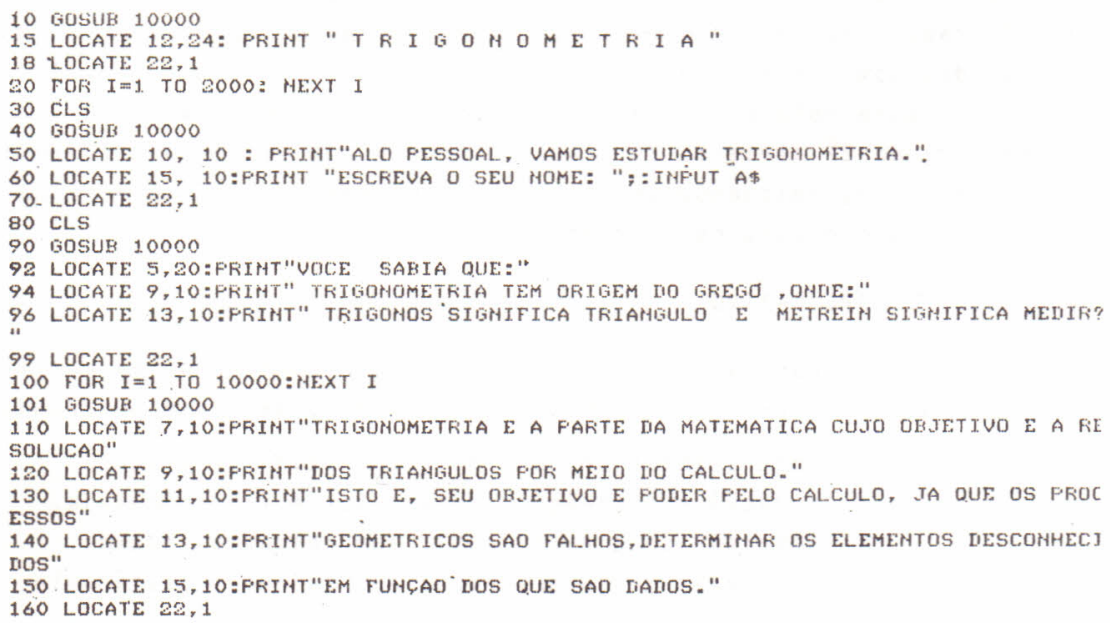


170 FOF I $=1$ TO 10000

180 HEXT I

190 CLS

200 GOSUR 10000

210 LOCATE 10,10:PRIHT"ESTE PROELEMA E DE GRAHDE IMPORTAHCIA, FOIS E FUHDAMEHTAL

220 LOCATE 12,10:FRIHT"HA HAUEGACAO MARITIMA OU AEREA E, HA LHGEHHARIA ESFECTALME MTE"

230 LOCATE 14,10:PRINT"EM TOPOGRAFIA."

240 LOCATE 22, 1:FOR I=1 TO 10000: HEXT I:CLS

250 GOSUB 10000

260 LOCATE 4,6:FRIHT"Voce podera escolher as seguintes opcioes, neste trabalho de. revisao:"

262 LOCATE 7,9:PRTHT"1) RAZOES TRIGOHOMETRICAS HUM TRIAHG. RETAHGULO."

264 LOCATE 9,9:PRINT" 2 ) RAZOES TRIGOHOMETRICAS HUM TRIAHG. QUALOUEK ."

266 LOCATE 11,9:PRINT"3) TRIGOMOMETRIA HA CIFCUHFEREHCIA."

268 LOCATE 13,9:FRIHT"4) CICLO TRIGOHOMETRICO."

270 LOCATE 17,9:PRIHT"5) TELA FIHAL

275 LOCATE 20,9:IHFUT" DIGITE SUA OPCAO : ";H

278 OH H EOSUB $500,3000,3610,4230,9800$

290 LOCATE 2E, $1:$ FOR I=1 TO $10000:$ HEXT I:CLS

500 GOSUB 10000

502 LOCATE 8,13:FRIMT"A SEGUIR UAMOS IHICIAR HOSSO TRABALHO DE FEUISAO,"

503 LOCATE 10,13:FRIHT"ESTUDAHIO FAZOES TFIGOHOMETRICAS HUM TRIAHGULO FE-"

504 LOCATE 12,13:FFINT"TAHGULO. OBSERUE A FIGURA A SEGUIF:"

505 LOCATE 22, 1 :FOR I=1 TO 5000: HEXT I:CL.S

506 G05UB 9860

508 GOSUE 10000

510 LOCATE 3,10:FFIAT"COHSIDEFAHIO O TFIAHGULOO FETAHGULO AEC DA TELA AHTERTOF, TE mos:"

5ZO LOCATE 5,12:PRIHT"AHGULO B=90 GFAUS"

530 LOCATE 7,12: FFIIHT"A+C=90"

540 LOCATE 9, 12 :PFIHT" $\mathrm{A}+\mathrm{H}+\mathrm{C}=130$ "

550 LOCATE 11,12:FRIHT"AH=CATETO OPOSTO AO AHISULO C"

560. LOCATE 13,12:PFINT"HC=CATETO ADIACEHTE AO AHGUULO C"

570 LOCATE 15, 12:FRIHT"HC=CATETO OFOSTO AO AHGULO A"

580 LOCATE 17, 12:FFIHT "AR=LATETTO ADJACEHTE AO AHTGULO A"

590 LOCATE 19,12:PFIHT"AC=HIPOTEHUSA"

600 LOCATE 21,10

610 PRIHT"PARA COHTIHUAR, LIGITE UMA TECLA ";:IHPUT A\$

620 GOSUB 10000

630 L.ECATE 6,10:FFIHT" VOCE TA FSTUDOU EM AULA DUE:"

"640 LOCATE 8,10:FFIHT"HUM TRIAHGULO RETAHGULO SAO VALIDAS AS SEGUIMTES RIELACOES: 650 LOCATE 9,10:FFIHT"SEHO DE UM AHGULO AGUDO E DAIO PELA RAZAO EHTRE O CATETO O POSTO"

660 LOCATE 10,10:PRIHT"AO AHGULO COHSTIERANO E A HIPOTENUSA."

670 LOCATE 12,10:PRIHT"COSSEHO IE UM AHGUL.O AGUDO E DADO FELA RAZAO EHTFE O CATE TO ADITH-"

G80 LOCATE 13,10:PRIHT"CEHTE AO AHGULO COHSIDERAIO E A HIPOTERIUSA."

690 LOCATE 15,10:FRIHT"TAHGENTE IIE UM AHGULO AGUIO E MALA PELA RAZAO EHTRE O CAT

ETO -OFOS-"

700 LOCATE 16,10:FFIHT"TO E CATETO ADTACEHTE AO AHGULO COHSIDERADO."

710 LOCATE 20,10:PRIHT"PARA COHTIHUAR, IIGITE UMA TECLA "; IHAUT A\$

715 GOSUB 9860

718 GOSUB 10000

720 LOSATE 6,10:PRINT"HO TRIAHGULO RETANGULO ABC DA HOSSA FIG. ,TEMOS:"

730 LOCATE 8,17:FRIHT" SEH A=BC/AC"

740 LOCATE 10,17: PRIHT" COS $A=A B / A C "$

750 LOCATE 12,17:FRIHT" TG $A=B C / A B^{\prime \prime}$

760 LOCATE 14,17: PRIHT" SEH C=AB/AC"

770 LOCATE 16,17: PRINT" COS $\mathrm{C}=\mathrm{BC} / \mathrm{AC} "$

780 LOCATE 18,17:PRIAT" TG C=AB/BC"

790 LOCATE 20,10:PRIHT"F'ARA COHTIHUAR, IIGITE UMA TECLA "; INAUU A\$

795 GOSUB 10000

800 LOCATE 4,8:PRINT"FARA FIXAF ESTAS RELACOES, RESOLUA OS SEGUIHTES EXERCTCIOS:" 810 LOCATE 8,8:FRIHT"1 JCOHSIDERAHDO O TRIAHGULO RET. ABC COM AB=3 BC=4 AC=5, CALC ULE:"

820 LOCATE 10,8:PRIHT"SEH A, COS. A, TG A, SEH C, COS C, TG C"

830 LOCATE 13,8:PRIHT"2 ICOHSIDERAHIO O TRIAHGULO RET. ABC COM AB=6 BC=4, CALCULE:

840 LOCATE 15;8:PRINT"SEH A, $\operatorname{COS}$ A,TG A,SEN C, COS C,TG C"

850 LOCATE 17,8:PRIHT" 3 ) CONSIDERAHDO O TRIAHG. RET. AEC COM AB=6 BC=4*2^1/2, CALCU LE:"

860 LOCATE 18,8:PRINT"SEN A, COS A,TG A,SEM C, COS C,TG C"

870 LOCATE 20,8:PRIHT"PARA COHTIHUAR, DIGITE UMA TECLA "; :-

880 GOSUA 10000

900 LOCATE 4,6:PRIHT"AGORA, UERIFIOUE SE VOCE ACERTOU OS EXERCICIOS PROPOSTOS."

910 LOCATE ?, 10:PRIHT". 1) SEH $A=0.8, \ldots$ COS $A=0.6$. TG $A=1.33 "$ 


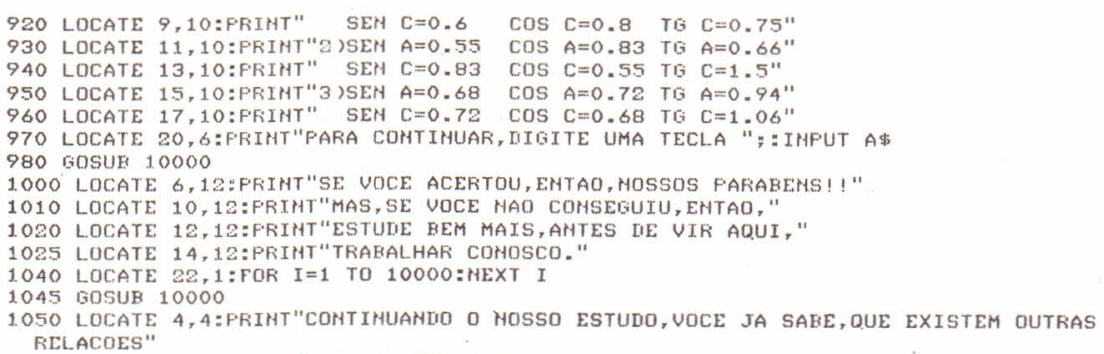

1060 LOCATE 6, 4:FFIHT"IMFORTAHTES OBTIDAS A PARTIR DE UM TRIAHG. RETAHGULO.EHTRE ELAS:"

1070 LOCATE 8, 4:FFIHT"A TAHGEHTE DE UM AHGULO AGUDO E DAIA PELA RAZAO EHTRE O SE HO E. O COSSEHO"

1080 LOCATE 10,4:PRIHT"IIESTE MESMO AHGULO."

1090 LOCATE 12, 4:FRIHT"ASSIM, HO TRIAHG.RETAHGULO ABC DO HOSSO ESTUNO,TEMOS:"

1100 LOCATE 14, 4:FFIHT"SEH $A=B C / A C$ COS $A=A B / A C$ TG $A=B C / A B "$

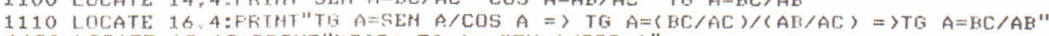

1120 LOCATE 18,19:FFIHT"LOGO: TG $A=S E H A / C O S A "$

1130 LOCATE 20,4: IHPUT"PARA COHTIHUAR, DIGITE UMA TECLA ";A\$

1140 G0SUR 10000

1150 LOCATE 3,4:FFIHT"AGQRA, EM SEU CADEFHO, VERIFIQUE QUE O MESMO ACOHTECE COM O AHTHULO AGURO C"

1160 LOCATE 5,4:FRIHT"ISTO E: TG C= SEH C / COS C"

1170 LOCATE 7;4:FFIHT"COMO COHSEQUEMCIA DO TEOREMA IE PITAGORAS, TEMOS OUTRA IMFO RTANTE RELA-"

1180 LOCATE 8, 4 :FRIHT"CAO:"

1190 LOCATE 10,4:PRIMT"HUM TRIAHG. RET.ABC, EXISTE UMA RELAÇAO FUMDAIEHTAL EHTKE

SEHO E COS -"

1200 LOCATE 11,4:FRIHT"SEHO DE UM AMGULO AGUDO, DADA POR:"

1210 LOCATE 13,10:PRIHT"(SEN A $)^{\wedge} 2+(\operatorname{COS~} A)^{\wedge} 2=1 "$

1220 LOCATE 15,4 :PRIHT"O MESMO, ACOHTECE COM O AHGULO C, ISTO E:"

1230 LOCATE 17, 10:PRIHT" (SEH C $)^{\wedge} 2+(\operatorname{COS~C})^{\wedge} 2=1^{\prime \prime}$

1240 LOCATE 20,4 I IHFUT"PARA COHTIMUAR, DIGITE UMA TECLA ";A"

1250 GOSUB 10000

1260 LOCATE 4, 4:PRIHT"AGORA, JA TEHDO REUISADO AS RELACOES AHTERIORES, FESOLUA OS

SEGUIHTES EXER-"

1270 LOCATE 5, 4:PFIHT"CICIOS:"

1280 LOCATE 9,4:FRIHT"1)DALO O VALOR DE SEN A=1/2, CALCULE: COS A E TG A"

1290 LOCATE 13,4:FRIHT"Z) IALO O UALOF DE TG C=3/4, CALCULE: SEN C E COS C"

1300 LDCATE 17,4:FRIHT"3) IIADO O VALOR DE COS A=5/6, CALCULE:SEN A E TE A"

1310 LOCATE 20,4: IHPUT"PARA COHTIHUAR, IIIGITE UMA TECLA ";A\$

1320 GOSUB 10000

1330 LOCATE 4,4:PRIHT"VAMOS VERIFICAR SE VOCE ACERTOU OF -XERCICIOS PROPOSTOS."

1340 LOCATE 7,12 :FRIHT" 1 ) COS $A=0.866$ E TG $A=0.577^{\prime \prime}$

1350 LOCATE 10, $12:$ PRIHT" 2 )SEH $C=0.6$ E COS $C=0.8$ "

1360 LOCATE 13,12:PRIHT" 3 ) SEN $A=0.55$ E TG $A=0.66$ "

1370 LOCATE 15,4:FRIHT"SE VOCE ACERTOU, EHTAQ, PARABENS!!!"

1380 LOCATE 17,4:PRIHT"EM CASO COHTRARIO, ESTUDE BEM AS RELAÇOES DADAS E REFAC,A O

S EXERCICIOS."

1390 LOCATE 20,4 : IHPUT"FARA COHTIHUAR, DIGITE UMA TECLA ";A\$

1400 GOSUB 10000

1410 LOCATE 4,4:FRIHT"UOCE JA ESTUDOU EM AULA, UMA TABELA DE UALORES MUITO IMPORT AHTE."

1420 LOCATE 6, 4:PRIHT"ESTA TAEELA,E OBTIDA PELA AFLICACAO DO TEOREMA DE PITAGORA

5, COHSI LERAHDO"

1430 LOCATE 8,4:PFIHT"UM TFIAHG.EQUILATERO COM A MEDIDA DE CADA AHGULO IHTEFIHO D

E 60 GRAUS"

1440 LOCATE 10,4:PFIHT"E UM TRIAHG.RET. TSOSCELES, OHDE CADA AHGULO AGUDO MEDE 45

ERAUS."

1450 LOCATE 12,4:PRIHT"OS AHGULOS QUE SAEM DESTA FELACAO SAO: 30,45 E. 60 GRAUS E

RECEBEM 0 "

1460 LOCATE 14, 4:PRIHT"MOME DE AHGULOS HOTAUEIS,FOIS, SAO MUITO UTILIZADOS HA TRI

GOHOMETRIA."

1470 LOCATE 16,4:PRIHT"OBSERUE COM MUITÄ ATEHÇAO, A TABELA DA PROXIMA TELA."

1480 LOCATE 20,4 : IHFUT"PARA COHTIHUAR, DIGITE UMA TECLA "; A

1490 GOSUB 10000

1495 LOCATE 5,14:FOR I=1 TO 40:FRIHT"-";:HEXT I

1500 LOCATE 6,14:PRIHT" $30 \quad 45$

1505 LOCATE 7,14:FOR I=1 TO 40:PRIHT"-";

1506 MEXT I

1510 LOCATE 8,14:FRIHT"SEH

1520 LOCATE 10,14:PRIHT"COS

1530 LOCATE 12,14:PRIHT"TG

$1 / 2$

$\operatorname{SOR}(3) / 2$

$\operatorname{SQR}(3) / 3$
$\operatorname{SQR}(2) / 2$ $\operatorname{SQR}(2) / 2$

1.

\section{$60^{\prime \prime}$}

$\operatorname{SQR}(3) / 2^{\prime \prime}$

$1 / 2^{\prime \prime}$

$\operatorname{son}(3) "$ 
1535 LOCATE 13,14:FOR I=1 TO 40:PRINT"-";:HEXT I

1540 LOCATE 20,6:IHPUT"PARA COHTIHUAR, DIGITE UMA TECLA ";A\$

1550 GOSUB 10000

1560 LOCATE 4,4:FRIHT"AGORA,RESOLUA OS SEGUIHTES EXERCICIOS:"

1570 LOCATE 6,4:PRIHT"1)A ALTURA DE UM TRIAHG. EQUILATERO MEDE 4 CM.CALCULE:"

1580 LOCATE 8,4:PRIHT"A)A MEDIDA DO LADO DO TRIAHG. B )A AREA DO TRIAMG. (AREA=BA

SE*AL.T. )"

1590 LOCATE 11,4:FRIHT"Z JCALCULE A ALTURA DE UM TRIAHG. EQUILATERO QUE TEM 1O CM DE LADO."

1600 LQCATE 14,4:FRIHT"3 UUM MOUEL, HUMA UIA IHCLIHAIIA IIE 30 GRAUS EM RELAÇAO A HO RIZOHITAL,"

1610 LOCATE 16,4:PRIMT"QUAMTO SOBE UERTICALMEHTE AO PERCORRER 2O METROS?"

1620 LOCATE 20,4: IHPUT"PARA COHTIHUAR, DIGITE UMA TECLA ";A\$

1630 GOSUB 10000

1660 LOCATE. 4,4:PRIHT"UERIFIQUE SE VOCE ACERTOU OS EXERCICIOS PROPOSTOS."

1670 LOCATE 7, 4:PFIHT"1) a) SEM $60=4 / \mathrm{L}$ COMO SEH $60=$ SQR(3)/2 EHTAO:"

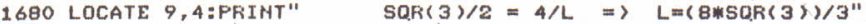

1690 LOCATE $11,4:$ PRIHT" b) $A=L * H \quad \Rightarrow A=(8 * \operatorname{SOR}(3)) / 3 * 4=(A=(32 * \operatorname{SOR}(3)) / 3 "$

1700 LOCATE 14,4:PRIHT" 2 ) HO TRIAHG. EQUILATERO CADA AHGULO IHTERHO MEDE GO GRAUS

1710 LOCATE $16,4: P R I H T "$ SEH $60=H / 10 \Rightarrow H=(10 \% \operatorname{SOR}(3)) / 2=5$ 米QR(3)"

1720 LOCATE 19,4 :PRINT" 3 ) AC $=20 \mathrm{E} B C=X \Rightarrow S E H \quad 30=X / 20 \Rightarrow 1 / 2=X / 20 \Rightarrow \quad X=10^{\prime \prime}$

1730 LOCATE 21,4 IMPUT"PARA COHTIMUAR, DIGITE UMA TECLA ", A\$

1740 GOSUB 10000

1750 LOCATE 4,4:PRIHT" SEGUIHDO A SEQUEHCIA IEE EXERCICIOS, RESOLUA:"

1760 LOCATE 6,4:PRIHT"4 INUM TRIAMG.RETAHGULO,A HIPQTENUSA MEDE 3A E OS CATETOS M

EDEM A E"

1770 LOCATE 7,4:PRIHT" 2*AWSOR(2).CALCULE:"

1780 LOCATE 9,4:PRIHT"A)A TAHGEHTE DO AHGULO OPOSTO AO MEMOR CATETO."

1790 LOCATE 11,4:PRIHT"B JO SEHO DO AMGULO OPOSTO AO MAIOR CATETO."

1800 LOCATE 14,4:PRIHT"5)UMA ESCADA APOIADA EM UMA PAREDE, NUM POHTO OISTANTE 4M

DO SOLO,FOR-"

1810 LOCATE 16,4:PRINT."MA COM ESSA PAREDE UM AMGULO DE 60 GRAUS.QUAL E O COMPRIM

EHTO DA ESCA-"

1820 LOCATE 18,4:PRIHT"DA EM METROS?(SEN60=0.86;COS60=0.50, T660=1.73)"

1830 LOCATE 21,4 : IHPUT"PARA COHTIHUAR, DIGITE UMA TECLA ";A\$

1840 GOSUB 10000

1870 LOCATE 4,4:PRIMT"ÜERIFIQUE SE SEUS RESULTADOS ESTAO CORRETOS."

1880 LDCATE 7,4:PRIHT"4) A) TG C=CAT.OP./CAT.ADJ. $\Rightarrow$ TG $C=A /(2 * A * S Q R(2))=$ TG C

$=\operatorname{SQR}(2) / 4^{\prime \prime}$

1890 LOCATE 9,4:PRIHT" B) SEM A=CAT.OP./HIP. $\Rightarrow S E M A=(2 * A$ * SQR $(2)) / 3$ *A $=S$ SEM

$A=(2) \operatorname{SQR}(2)) / 3^{\prime \prime}$

1900 LOCATE $14,4:$ PRINT"5) $\operatorname{COS} 60=$ CAT.ADJ./HIP. $\Rightarrow 1 / 2=4 / X=X=8 "$

1910 LOCATE 20,4: IMPUT"PARA COHTIHUAR, DIGITE UMA TECLA ";As

1920 GOSUR 10000

1.930 LOCATE 4,4:FRIMT"SEGUIMDO A SEQUEHCIA DE EXERCICIOS, RESOLVA:"

1940 LOCATE 6,4:PRIMT"6 JUM GUARDA FLORESTAL,POSTADO MUMA TORRE DE 2OM MO TOPO DE UMA COLINA DE"

1950 LOCATE 7,4:PRIHT"5OOM DE ALTURA, VE 0 IHICIO DE UM INCEMDIO MUMA DIRECAO QUE FORMA COM A "

1960 LOCATE 8,4:PRIMT"HORIZOHTAL UM AHGULO DE 17 GRAUS.A QUE DISTANCIA APROXIMAD A DA COLIHA "

1970 LOCATE 9,4:PRIHT"ESTA O FOGO? (SEN 17=0.29;COS 17=0.95;TG 17=0.30)"

1980 LOCATE 13,4:PRIHT"7 HHU EXERCICIO DE TIRO,O ALVO SE EHCOHTRA MUMA PAREDE CU

JA BASE ESTA

1990 LOCATE 14,4:PRIHT"SITUADA A 82M DO ATIRADOR.SABEHDO QUE O ATIRADOR UE O ALU O SOB UM AH-"

2000 LDCATE 15,4:PRIMT"GULO DE 12 GRAUS EM RELAÇO A HORIZONTAL, CALCULE A QUE DI STAHCIA DO CHAO"

2010 LOCATE 16,4:PRIHT"ESTA O ALUO?(SEH 12=0.20, COS 12=0.97; TG 12=0.21)"

2020 LOCATE 20,4:IHPUT"PARA COHTIHUAR, DIGITE UMA TECLA ";A\$

2040 GOSÜB 10000

2060 LOCATE 4, 4:PRIHT"UERIFIOUE. SE VOCE ACERTOU SEUS EXERCICIOS."

2070 LOCATE 7,4:PRIHT"6) $T G 17=$ CAT,OP/CAT.ADJ. $\Rightarrow$ TG $17=520 / X=10.30=520 / X$

2080 LOCATE 14,4:PRIHT"7) TG $12=$ CAT.OP./CAT.ADJ. $\Rightarrow$ TG $12=H / 82=10.21=H / 8$ $2=\mathrm{H}=17.22^{\prime \prime}$

2090 LOCATE 20, 4: IHPUT"PARA COHTIHUAR, DIGITE UMA TECLA "; A\$

2095 GOTO 250

3000 GOSUB 10000

3005 LOCATE 8,12:PRIHT"AGOFA, VAMOS REUISAR TRIAMGULOS QUAISOUER,"

3008 LOCATE 12,12:FRINT"OBSERUE 0 TRIAHGULOO A SEGUIR:"

3010 LOCATE 22, 1 : FOR I=1 TO $6000: \mathrm{HEXT}$ I :CLS

3015 GOSUB 11000

3020 GOSUB 10000

3030 LOCATE 6, 4:FFIHT"VOCE JA SABE, QUE A PARTIF DOS COHCEITOS DE SEHO E COSSEMO, 
EXISTEM DUAS"

3040 LOCATE 8,4:FRINT"IMFORTAHTES RELAÇOES EHTRE DS LADOS DE UM TRIAHG. QUALQUEF ."

3050 LOCATE 10,4:PFIHT"LEI DOS COSSEHOS:HUM TFIAHG.QUALQUER,O QUADRADO DE UM LAD O E IGUAL A SO-"

3060 LOCATE 11,4:PRIHT"MA DOS QUADRADOS DOS OUTROS DOIS LADOS MEHOS DUAS UEZES. 0 PRODUTO DESTES"

3070 LOCATE 12,4:PRIHT"IIOIS LADOS FELO COSSEHO DO AHGULO FORMADO FOR ELES."

3080 LOCATE 15, 4:PRIHT"LEI DOS SEHOS:A RAZAO EHTRE A MEDIDA DE UM LADO E O SEHO

IOO AHGULO OFOS-"

3070 LOCATE 16,4:PRIHT"TO E COHSTAHTE HUM MESMO TRIAHGULO."

3095 LOCATE 18,4:PRITHT"ASSIM, CONSIDERAHDO O TRIAHG. QUALOUER ABC IAA PROXIMA TELA,

TEMOS:"

3098 LOCATE 21,4 I IHFUT"PARA COHTIMUAR, DIGITE UMA TECLA ";A\$

3099 GOSUR 11000

3100 GOSUB 10000

3300 LOCATE 4, 4:FRIHT"HO TRTJAHG. QUALQUER ABC, TEMOS:"

3310 LOCATE 6, 4: PRIHT"OS LAIOS: $A B=C \quad B C=A \quad A C=B$ '

3320 LOCATE 8, 4:PRIHT"ASSIM,FODEMOS ESCREUER:"

3330 LOCATE 11,4:PRIHT"LEI DOS COSSEHOS: A $2=B^{\circ} 2+C \cdots 2-2 * B * C * C O S A "$

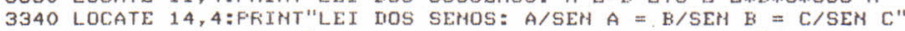

3350 LOCATE 20,4: IHPUT"PARA COHTIHUAR, IIGITE UMA TECLA "; A

3360 GOSUB 10000

3370 LOCATE 4, A:PRIHT"AQORA, QUE VOCE JA RECORIOU ESTAS DUAS IMPORTAMTES RELACOES ,RESOLUA OS SE-"

3380 LOCATE 5, 4:PRIHT"QUINTES EXERCICIOS:"

3390 LOCATE 7, 4: PRIHT"1 )HUM TRIAHG. ABC, A=SQR(2), B=SQR( 3) E A=45 GRAUS. CALCULE OS AHEULOS B E. C."

3400 LOCATE 9, 4:PRIHT"Z)HUM TRIAHG. ABC, $B=4, C=S Q F(3)$ E $A=30$ GRAUS.CALCULAR O LADO A."

3410 LOCATE 11,4:PRIHT"3) SEHDO DADOS OS AHGULOS A=80 GRAUS E B=4O GRAUS E 0 LADO

$\mathrm{C}=6$ DE UM TRI $-"$

3420 LOCATE 12, 4:PRIHT"AMG. ABC, DETERMINE OS DUTROS DOIS LADOS."

3430 LOCATE 14,4:PRIMT"4 INOIS HAUIOS IISTAHTES UM DO OUTRO, EHXERGAM UM MESMO FAR OL. SOB AHGU-"

3440 LOCATE 15,4:PRIMT"LOS DE 30 E 45 GRAUS. A DUE DISTAHCIA DO FAROL ESTA CADA H AUIO?"

3450 LOCATE 17, 4:FFIMT"5)OS LADOS DE UM PARALELOGRAMO MEDEM 6CM E SOR( 2 )CM E FOR MAM UM AHGULO "

3460 LOCATE 18,4:FRIHT"DE 45 GFAUS.CALCULAR AS MEDIDAS DAS DIAGOHAIS DO PARALELO GRAMO."

3465 LOCATE 21,4: IHPUT"PAFA COHTIHUAF, DIGITE UMA TECLA ";A\$

3470 GOSUB 10000

3500 LOCATE 4, 4:PRIHT"UERIFIQUE SE VOCE ACERTOU TODOS OS EXERCICIOS."

3510 LOCATE 6, 4:FRIHT"1)A/SEH $A=B / S E H ~ E \Rightarrow S Q F(2) / S E H ~ 45=5 Q R(3) / S E H A=>S E M$ $B=\operatorname{SQR}(3) / 2^{\prime \prime}$

3520 LDCATE 7, 4:PRIHT" $B=60$ GRAUS COMO: $A+B+C=180 \Rightarrow C=180-45-60 \Rightarrow C=756 R A$ US"

3530 LQCATE 9, 4:PRIHT"Z) $A^{\wedge} 2=B^{\wedge} 2+C^{\wedge} 2-2 * B *$ * $*$ COS $A \Rightarrow A=\operatorname{SOR}(7) "$

3540 LOCATE $11,4:$ FFIHT" 3 ) COMO $A+B+C=180 \Rightarrow C=60^{4}$

3550 LOCATE 12, 4:PRIHT" EHTAO:A/SEH $80=B / S E H ~ 40=6 /$ SEH $60 \Rightarrow$ A $A=6.76 \mathrm{E} \quad \mathrm{B}=4.4 "$

3560 LOCATE 14,4:FRIHT"4) $A+B+C=180 \Rightarrow C=105 \Rightarrow A / S E M \quad 30=E / S E M \quad 45=56 / S E M 105=>$

$A=29,1$ E $B=41,24^{\prime \prime}$

3570 LOCATE $14,4:$ PRINT" 4 ) $A+B+C+D=360 \Rightarrow A=C=45 \quad E \quad B=\left[=135^{\prime \prime}\right.$

3580 LOCATE $16,4:$ PRIHT"S $)\left[11^{\wedge}=(\operatorname{SOF}(2))^{\wedge} 2+6^{\wedge} 2-2 * S O R(2) * 6\right.$ * M"

3590 LOCATE 17,4:PRIMT" D2`2=6^2+(SQR(2)) $2-2 * 6 * S Q R(2) * \operatorname{COS} 135=$ D2=5*SQR(2) CM"

3600 LUCATE 20,4: IHPUT"PARA COHTIHUAR, DIGITE UMA TECLA ";A\$

3605 G0T0 250

3610 G0SUE 10000

3620 LOCATE 4, 4:PRIHT"COHTIHUAHDO O HOSSO ESTUDO, VAMOS FEUISAR AGORA, A TFIGOHOME

TFIIA HA CIFI-"

3625 L.OCATE 5, 4:PRINT"CUHFEREHCIA."

3630 LOCATE 7, 4:FFIHT"VOCE JA SAEE QUE A CIFCUHFEREHCIA E O COHJUHTO DE TODOS OS PONTOS DE

3635 LOCATE 8̈, 4:FRIHT"UM PLAHO QUE ESTAO A UMA MESMA TIYSTAMCIA R DE UM POHTO DAL 0 0."

3640 LOCATE 10,4:FRIHT"VOCE JA SABE TAMBEM, QUE RETIFICAHLO-SE A CIRCUHFEREHCIA, TEMOS DUE"

3645 LOCATE 11, A:FRIHT"SEU COMPRIMEHTO E:C = 2 \% 3.14 * F.CHAMAREMOS AQUI, R O R AIO DA CIRC."

3650 LOCATE 12, 4 :PRIHT" E, DE O SEU CENTRO."

3655 LOCATE 14,4:PRIHT"PARA SEGUIR O HOSSO ESTUDO, E HECESSARIO O COHHECIMEHTO SO BRE AFICOS E"

3660 LOCATE 15, 4:PRIHT"AHBULOS DE UMA CIRCUHFEFEHCIA E SUAS MEDIDAS."

3665 LOCATE 17,4:PRIHT"VAMOS COHSIIERAR AQUI, A CIRCUHFEREHCIA SITUALA HA PROXIMA TELA"

TELA"
3670 LOCATE 21,4: IHPUT"PARA COMTIHUAR, DIGITE UMA TECLA ";A\$ 
3675 EOSUE 10000

3677 GOSUE 12000

3800 LOCATE 4, 4:FRIHT"TEMOS QUE DOIS FOHTOS DISTIHTOS A E B,SITUADOS SOBRE A CIR C. , IIUIDEM"

3805 LDCATE 5, 4:PRIHT"ESTA EM DUAS FAFTES."

3810 LOCATE 7,4:PRIHT"CALA UMA IIESTAS PARTES, IHCLUIHDO A E B,RECEBEM D HOME IIE A RCO IE CIR-"

3815 LDCATE 8, 4:FRIHT"CUHFEREHCIA AB."

3820 LOCATE 10,4:FRIHT"EM FARTICULAR, SE A COIHCIDE COM B, EHTAO, TEMOS:O AFCO HULO (QUE E UM FTO)"

3825 LQCATE 11,4:PRIMT "E O ARCO IIE UMA VOLTA(QUE E A CIRCUNFEREHCIA)"

3830 LOCATE 13,4:FRIHT"FARA MEDIR O COMFFIMEHTO NE UM ARCO AB, USAREMOS O GRAU E

O RADIAHO CO-"

3835 LOCATE 14,4:FRINT"MO UHIMADES IE MEDIDA."

3840 LOCATE 16,4:FRIPT" GRAU E UM ARCO UHTTARIO IGUAL A $1 / 360$ DA CIRC. QUE COHT

EM 0 AFCO AB."

3845 LOCATE 18,4:FFIHT" FADIAHO(RAD) E UM ARCO UHITARIO, CUJO COMPRIMENTO E IGUAL AO RAIO IIA "

3850 LOCATE 19,4:PRIHT"CIRCUAFEFEHCIA QUE COHTEM 0 ARCO AE,"

3855 LOCATE 21, 4 : IHFUT"FAFA COHTUHUAF, IIGITE UMA TECLA "; $\$$

3860 DOSUR 10000

3870 LOCATE 4,4 ,FFIHT"E IMPORTANTE SABEF DUE:"

3875 LOCATE 6,4:FRIHT"A CIFC. TEM 360 GRAUS, MAS, QUE O COMFFIMEHTO IIESTA EM RAII E OBYTRO ATRA-"

3880 LOCATE 7, 4:FFIHT"UES DE UMA COHSTFUC,AO GEOMETFICA,A QUAL UOCE DEVE TEF ESTU DALO EM AULA."

3885 LOCATE 9, 4 :FFIHT"ASSIM ESTAEELECEMOS QUE:"

3890 LOCATE 12, 4:PRIHT"UMA CIRC. MEIE: 360 GFAUS $\Leftrightarrow 2$ * 3.14 FAD"

3895 LOCATE 15, 4FRIHT"MEIA CIFIC.MEDE: 180 GFAUS $\Leftrightarrow 3.14$ FAD"

3900 LOCATE 18,4:PFIHT"AIHLAA: MEIILA DO AFCO ABSEM RAD) = COMF. DO AFCO AB/COMF. DO RATO DA CIFC."

3905 LOCATE 21,4:IHPUT"FAFA COHTIHUAF, DIGITE UMA TECLA "; A\$

3910 GOSUB 10000

3920 LOCATE 4, 4:FRIHT"DADA A CIRC, IDE CEHTRO O E IOIS POHTOS A E B SCBFE ESTA,TE MOS QUE O Ait-"

3930 LOCATE $5,4: F R I H T " G U L O$ FOFMATO FELOS SEGMEHTOS OA E OB COM UERTTCE PU CEHTRO D DESTA CIRC."

3940 LOCATE 6, 4 :FFIHT"RECEFE O HOME DE AHIGULO CEPTTRAL. LOGO:"

3950 LOCATE 8,22:PFIHT"AOE = AHGULO CEHTRAL"

3960 LOCATE 10,4:FFIHT"IIESTA MAHEIFA, A CAIAA ARCO AB CORFESPOHDE UM UHICO AHEULO CEHTFIAL E. A"

3970 LOCATE 11,4:FFIHT"CAMA AHIUULO CEPTRAL COFFESFOHIE UM UPICO AFCO AB "

3980 LOCATE 13,4:FFIHT"COHUEHCIOHA-SE, EHTAO, QUE A UM ARCO UHITARIO CORFESFOHIE U $M$ AIIEULLO UHI -"

3985 LOCATE 14,4:PRIHT"TAFIO."

3990 LOCATE 16,4:FFIHT"ASSIM:O AFCO. AB E O AHIGULO CEHTFAL AOB COFFESFOMIEHTE PAS SAM A TEF A"

3995 LOCATE 17, 4:FFIMT"MESMA MEIIIA."

4000 LOCATE 19, 4:FFIHT"LOEO: MED(AOB)EM FIAD = COMF,IIO ARCO AB , COMP. DO FIAIO DA

CIFiC."

4005 LOCATE 21, 4: IHFUT"PAFA COHTIMUAF, IIITTE UMA TECLA ";A\$

4010 GOSUB 10000

4015 LOCATE 4, 4:FRIHT"AGOFA, FESOLUA OS SEEUTMTES EXEFCICIOS:"

4020 LOCATE 6, 4:FFIHT"1 DDARO O ARCO DE (3*3.14)/4 RAD, DETEFMIHE A MENIDA EQUIUAL

EHTE FM IFFAUS."

4025 LOCATE 8,4:FFIHT"2 JCOHUEFTA FAFIA FAI O ARCO DE 315 GRAUS."

4030 LOCATE 10,4:FFIHT"3 IEM CARA CASO A SEGUIF, SAO RIADOS O COMF. L IOO ARCO AB E D RAIO FI IIA"

4035 LOCATE 11,4:FFIHT"CIFIC. CALCULE. A MEDIDA DO AFICO EM RAD."

4040 LOCATE 13,18:FFIIHT"A) $L=0.5 \mathrm{M} \quad \mathrm{E} \quad \mathrm{R}=0.25 \mathrm{M}$ "

4043 LOCATE $15,18:$ FFIIHT "B) $L=2 \mathrm{CM} \quad \mathrm{E} \quad \mathrm{F}=0.04 \mathrm{M}$ "

4045 LOCATE 1?, $18: F$ FFIHT" "C) $\mathrm{L}=0.105 \mathrm{CM} \quad \mathrm{E} \quad \mathrm{R}=0.42 \mathrm{CM}$

4050 LOCATE 21, 4:IPAUT"PAFA COHTIHUAR, DIEITE UMA TECLA ";A\$

4055 GOSUE 10000

4070 LOCATE 4, A:FFINT"UEFTFIOUE SE VOCE ACEFTOU OS EXEFCICTOS FFOFOSTOS:"

4075 LOCATE 7.4:FRIHT" 1) COMO 3.14 KAI $\Leftrightarrow 180$ GRAUS, EHTAO: $X=135$ GFAUS"

4080 LOCATE 10,4:FFIHT" 2 ) COMO 3.14 FAD $\Leftrightarrow 180$ GRAUS, EPTAO: $x=2.35$ FAD"

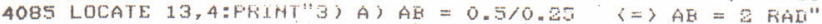

4090 LOCATE 15,4 FFIHT" $^{\circ}$ B) $A B=0.02 / 0.04 \Leftrightarrow A B=0.5$ FAL"

4095 LOCATE 17,4 "FFITHT" C) $A B=0.105 / 0.42\langle=>$ AE $=0.25$ FAII"

4098 L.OCATE 21,4:IHFUT"PAFA COHTIHUAF, DIGITE UMA TECLA ";Aक

4100 G0SUB 10000

4110 LOCATE 2, 4:FFIHT"COHTIHUAHIIO OS EXEFCICIOS, RESOLUA:"

4115 LOCATE 4, 4:FFIIHT"A) COHUERTA FARA FAD OS SEQUTHTES ARCOS:'

4120 LOCATE 6,4:FFIIHT"A) 22 GFAUS E 30 MIHUTOS E) 31 GRAUS 15 MIHUTOS E 45 SEG UHHOS. (LEM-"

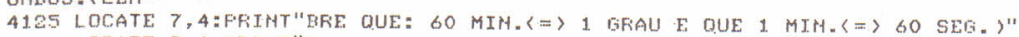

4130 LOCATE 9, 4 :PRIHT"S ) COHUERTA EM GRAUS O ARCO IIE 1 RAIIT"*פ."

4135 LOCATE 1.1,4:FRIHT" 6 DOETEFMIHE O MEHOR AHGULO FOFMADIO PELOS FOPTEIROS IIE, UM 
FELOGIO DUAH-"

4140 LOCATE 12, 4:FRIHT"DO ESTE MARCA 12 HORAS E 15 MIHUTOS. IOR DE UM RELO-"

4145 LOCATE 13,4:FRIHT"GIO ESTAO COLOCAIOS EM PTOS. QUE IIUIDEM A CIRC.EM 12 PART ES IGUAIS, OHDE"

4150 LOÇATE 14, 4:FRIHT"CADA PARTE MEDE 30 GRAUS.ASSIM, QUAHIOU O POHTEIRO MAIOR DA 1 VOLTA"

4155 LOCATE 15,4:PRIHT"COMFLETA O MEHOF SE DESLOCA IIE 30 GRAUS.)"

4160 LOCATE 17,4:FRIHT'7 IDETERMIHE O MEHOR AHGULO FORMADO FELOS POHTEIROS DE UM RELQGJO QUAAH-"

4165 LOCATE 18,4:FRIMT"IO ESTE MARCA 5 HORAS E 55 MIHUTOS."

4170 LOCATE 21,4: IHPUT"PARA COHTIHUAR, DIGITE UMA TECLA ";A\$

4175 GDSUB 10000

4190 LOCATE 4,4:PRIHT"UERIFIOUE SE VOCE ACERTOU OS EXERCICIOS PROPOSTOS:"

A195 LOCATE 6,4:FFIHT"4) A) IEUEMOS USAR A EOUIUALEHCIA: 3.14 FAI 〈= $10800 \mathrm{MIN}$." 4198 LOCATE $8,4:$ PFIHT" LOGO: $X=3.14 / 8$ RAD"

4200 LOCATE 10,4"FRIHT" B USAHIO A EOUIUALEHCIA:3.14 RAD〈= 648000 SE்"GUTHOS"

4205 LOCATE 12,4:PRIHT" TEMOS QUE: $X=0.54$ RAL"

4210 LOCATE 14,4:FRIHT"5) $x=57$ GRAUS 19 MIH. 20 SEG."

4215 LOCATE 16,4 :PRIHT" 6 ) $X=82.5$ GRAUS" .

4220 LOCATE 18,4 : PRIHT"7) $x=152.5$ GRAUS"

4225 LOCATE 21, 4: IHFUT"PAFA COHTIHUAR, DIGITE UMA TECLA ";A\$

4228 GOTO 250

4230 GOSUB 10000

4235 LOCATE 4, 4:PRIHT"AGORA, VAMOS REUTSAF O CICLO TRIGOHOMETFICO."

4240 LOCATE 6, 4:FRIHT"VOCE TA ESTUDOU, QUE FECEEE O HOME IE CICLO TRIGOHOMETFICO, A CIRCUPHFEFEH-"

4245 LOCATE 7, 4:PFIHT"CIA IE RAIO UHITARIO FI, A CARTESIAHO,"

4250 LOCATE 8, 4:FRIHT"CUJA ORIEHTAÇAO POSITIUA DOS ARCOS E DADA MO SEHTIIIO AHTIHORARIO."

4255 LOCATE 10,4:FRIHT"OS EIXOS COORDEMADOS X E Y DO SISTEMA CARTESIAHO, DIUIDEM. 0 CICLO EM $\hat{\beta}^{\prime \prime}$

4260 LOCATE $11,4:$ :PRIMT"QUAIRAHTES."

4265 LOCATE 13,4:FRIHT"OS EXTREMOS DESTES QUALRAHTES SAO OS FOHTOS: $(1,0),(0,1),($ $-1,0) \mathrm{E}(0,-1), "$

4270 LOCATE 1.4, 4:FFIHT"FOIS, O CICLO TEM FE=1."

4275 LOCATE $16,4:$ FEIHT"A OFIGEM DA CONTAGEM DOS ARCOS E O POHTO $(1,0) . "$

4280 LOCATE 18, 4:FFIHT"AIHIIA, DIZEMOS QUE UM ARCO PERTEHCE A UM DOS QUADRAHTES QU ANDO SUA EXTKE:-"

4285 LDCATE 19,4:PRITH"MIDADE ESTA HESSE QUARRAHTE."

4290 LOCATE 21,4:IHFUT"FAFA COHTIHUAF, DIGITE UMA TECLA ";A\$

4295 GOSUE 10000

4300 LOCATE 4, 4:FRIHT"AGORA, EM SEU CALERHO, IESEHHE O CICLO TRIG. E RESPOHDA EM O UE OUARIFAHTE

4305 LOCATE. 5,4:PRIHT"ESTA SITUANO CALA ARCO COHSIDEFADO."

4310 LOCATE 7,14:FRINT"1) AREO DE 135 GRAUS"

4315 LOCATE 9,14:PRIHT" 2 ) AFCO IIE 240 GRAUS"

4318 LOCATE 11,14:PRIHT"3) ARCO DE (7*3.14)/4 RAD"

4320 LOCATE 13,14:F'RIHT"4) ARCO. IE (7*3.14)/8 RAD"

4325 LOCATE 15,14:PRIHT"5) ARCO IIE (7*3.14)/6 RAD"

१330 LDCATE 17,14:FRTHT"6) ARCO DE 300 GRAUS"

4335 LOCATE 20,20: IHPUT"PARA COHTIHUAR, DIGITE UMA TECLA ";A\$

4340 IDOSUE 10000

4345 LOCATE 4,20:FRIHT" UERIFIOUE SUAS RESFOSTAS:"

4350 LOCATE 7,20:PRIMT"1) SEIUHLO QUADFAHTE"

4355 LOCATE 9,20:PRIHT"2) TERCEIFO QUADRAHTE"

4360 LOCATE 11,20:PRIHT"3) QUARTO QUAIRAHTE"

4365 LOCATE 13,20:PRIHT"4) SEGUHDO QUADRAHTE"

4370 LOCATE $15,20:$ PPIHT"5) TERCEIRO QUADRAHTE"

4375 LOCATE 17,20 :FRIHT"6) QUARTO QUALRAHTE"

4377 LOCATE 20,30: IHPUT"PARA COHTIHUAR, IIGITE UMA TECLA ";A\$

4380 GOSUB 10000

4385 LOCATE 4, 4:FRIHT"VOCE DEUE TAMHEM TER ESTUDADO, QUE E MUITO IMFORTAHTE DETEF MIHAR A QUE"

4390 LOCA,TE 5, 4:PRIHT"QUADRANTE FERTEHCE UM ARCO MAIOR DO QUE 3G GRAUS."

4395 LOCATE 7, 4: :PRIHT"ASSIM,FOR EXEMPLO, DADO UM ARCO AB DE 420 GRAUS, OUEREMOS SABER A QUE"

4400 LOCATE 8,4:PRIHT"QUDRAHTE FERTEHCE ESTE ARCO."

4405 LOCATE 10,4:PRIHT"PARA ISTO, TOMAMOS 420 E DIUIDIMOS POR 360, PARA SABERMOS D. UANTAS VOLTAS

4410 LOCATE 11,4:PRIHT"ELE PERCOFREU HA CIRC."

4415 LOCATE 13,4 :PRIHT"COMO, $420 / 360=1$ E TEM RESTO 60, SIGHIFICA QUE O ARCO IIEU 1 VOLTA E FERCOR-"

4420 LOCATE 14,4:PRIHT"REU MATS O AFCO CORRESFOHIEHTE A 60 GRAUS."

4425 LOCATE 16,4:PRIHT"ESTE VALOR DE 60 GRAUS(OUE PERTEMCE AO I QUADRATTE), RECEB E. 0 MOME DE"

4430 LOCATE 17,4:FRIHT"FRIMEIRA DETERMIHACAO POSITIUA DESTE ARCO AB=42O GRAUS."

4435 LOCATE 19,4:PRIMT"CHAMAREMOS AQUI, A I DETERMIHACAO POSITIUA IIE UM ARCO DE A 
$1 . "$

4440 LOCATE 21,4 : IHFUT"FARA COHTIHUAR,DIGITE UMA TECLA "; A\$

4445 GOSUB 10000

4450 LOCATE 4, 4:PRINT"VOCE DEUE LEMBRAR, QUE A I DETEFIMINAÇAO POSITIVA DE UM ARCO AB MEDE SEM-"

4455 LOCATE 5,4:PRIHT"PRE EHTRE: $0<A 1<360$ OU 0 < 1 < 2 * $3.14 "$

4460 LOCATE 7,4:PRIHT"CONSIDERAHDO AIHDA,O EXEMPLO DADO AHTERIORMEHTE, UEMOS QUE

O ARCO $A B=420 "$

4465 LOCATE 8,4:PRIHT"E O ARCO A1=60 TEM EXTREMIDADES COIHCIDEHTES E SAO CHAMADO

5 DE ARCOS

4470 LOCATE 9, 4:PRINT"COHGRUOS. GEHERAL IZAHDO:"

4480 LOCATE 12,4:PRIHT"ARCOS COHGRUOS, SAO ARCOS QUE TEM EXTREMIDADES COIHCIUEHTE 5."

4485 LOCATE 14,4:FRIHT"E VALIDA A SEQUIHRE FELAC,AO, QUAHDO A1 E AB SAO ARCOS COMI: Ruos:"

4490 LOCATE 16,4:FRIHT" $A B=A 1+2 * K * 3.14$ OU $A B=A 1+K * 360 "$

4495 LOCATE 18,4:FFINT"A1=I DET. FOSITIUA DO ARCO AB E K E O HUMERO DE VOLTAS QU

E DA O ARCO AB"

4498 LOCATE 21,4 : IHPUT"PARA COHTIHUAR, DIGITE UMA TECLA "; A\$

4500 GOSUE 10000

4505 LOCATE 4,20:PRIHT"RESOLUA OS EXERCICIOS SEGUIHTES:"

4510 LOCATE 6,20:PRIHT"EM CADA EXERCICIO DETERMIHE:"

4515 LOCATE 7, 20: FFIIHT" "A OO QUADRAHTE A QUE PEFTEHCE O AFCO DANO"

4520 LOCATE 8,20:PRIHT"B)ESCREVA O ARCO SOB A F'DRMA: AB = A1 + K * $360^{\prime \prime}$

4525 LOCATE 10,30:PFTHT"1) 1300 GFAUS"

4530 LOCATE 12,30:FRINT"Z) 450 GRAUS"

4535 LOCATE 14,30: F'FITH" 3 ) (21*3.14)/4"

4540 LOCATE 16,30:PFIIHT"4) (17*3.14)/6"

4545 LOCATE 18,30:PRIHT"S) -900 GRAUS"

4550 LOCATE 20,30:PFIHT"6) -1530 GRAUS"

4555 LOCATE 21, 22: IHPUT"PARA COHTIHUAR, DIGITE UMA TECLA ";A\$

4560 GOSUB 10000

4580 LOCATE 4,18:PRIHT "UERIFIQUE SUAS RESFOSTAS."

4585 LOCATE 6, 10:PFIHT"1) A )III QUADRAHTE B $1300=220+3 * 360^{\prime \prime}$

4590 LOCATE 8,10:PRINT"2) A) I QUADRAHTE B $450=90+1 * 360^{\prime \prime}$

4595 LOCATE 10,10:PFIHT"3) A)III DUADRAHTE B)(21*3.14)/4=(5*3.14)/4+2*2*3.14"

4600 LOCATE 12, 10:PFIHT"4) A) II QUAARAHTE B $(17 * 3.14) / 6=(5 * 3.14) / 6+1 * 2 * 3.14 "$

4605 LOCATE 14,10 : PRIHT"5) A)III QUALRAHTE B) $-900=-180-2 * 360^{\circ}$

4610 LOCATE 16,10:FRINT"6) A)III QUADRAHTE B) $-1530=-90-4 * 360^{\circ}$

4615 LOCATE 20,10: IHFUT"PARA COHTIHUAR, DIGITE UMA TECLA ";A\$

4618 GOTO 250

4620 CLS

9800 GOSUB 10000

9805 LOCATE 6,20:PRIHT A\$

9810 LOCATE 10,15:PRIHT"FOI UM FRAZER ESTUDAR COM VOCE."

9815 LOCATE 13,15:PRIHT"VOLTE HA PROXIMA SEMAHA... TCHAU..."

9820 LOCATE 22,1

9825 FOR I=1 TO $5000: H E X T$ I

9850 ENI

9860 REM TRIANGULO

$9870 \mathrm{CLS}$

9880 SCREEH 1: COLOR 0,1

$9890 \operatorname{LIHE}(100,100)-(180,100)$

$9895 \operatorname{LIHE}(180,100)-(180,50)$

$9900 \operatorname{LIHE}(100,100)-(160,50)$

9905 LOCATE 13,12:PRIHT" $A$ "

9910 LÖCATE 13,24:PRIINT"B"

9915 LOCATE 7,24:FRIHT"C"

9920 FOR I=1 TO 10000 :HEXT I

9930 SCREEH

9940 . WIDTH 80

9950 RETURH

10000 CLS

10010 FOR $I=1$ rO 77

10020 FFIIHT "*";

10030 HEXT I

10040 FOR I=1 TO 21

10050 PRINT "*": LOCATE I,78 :PRIHT "*"

10060 HEXT I

10070 FOR $I=1 \quad$ TO 78

10080 PRIHT "来";

10090 HEXT I

10110 RETUFH

11000 REM TRIAHGULO QUALOUER

11010 CLS

11020 SCREEH 1 : COLOF 0,1

$11030 \operatorname{LIHE}(100,100)-(180,100)$

11040 LIME $(130,100)-(140,50)$

$11050 \operatorname{LIHE}(100,100)-(140,50)$ 
11060 LQCATE $13,12:$ FFTHT "A"

11070 LOCATE 13,24:FFINT "B"

11080 LOCATE 6,19:FRIHT"C"

11085 FOF $I=1$ TO 5000 :HEXT I

11090 SCREEH

11100 WIDTH 80

11110 FETURH

12000 FEM CIFCULO

12010 CLS:SCFEEH 1 : KEY OFF

12020 COLOR 1,0

$12030 \operatorname{LIHE}(160,0)-(160,200)$

12040 L.IME $(0,100)-(320,100)$

12050 CIRCLE $(160,100), 60,2$

12060 L.IHE $(160,100)-(200,62), 2$

12070 LIHE $(200,62)-(200,100), 2$

12080 LOCATE 14,22:PFIHT"COSSEHO"

12090 LOCATE 11,27:PRIHT "SETU"

12100 FOF $I=1$ TO $6000: H E X T$ I

12110 SCREEH O

12120 WIDTH 80

12130 FETUFit 